\title{
El profesionalismo en la práctica médica actual
}

\author{
Professionalism in current medical practice
}

\section{José María Maya ${ }^{1}$}

1. Médico, Magíster en Dirección Universitaria, Magíster en Salud Pública. Rector, Universidad CES, Medellín, Colombia

\section{RESUMEN}

Se presenta la concepción moderna del profesionalismo y su aplicación al ejercicio de la medicina. A partir del proyecto de profesionalismo médico liderado por importantes asociaciones estadounidenses y europeas de medicina, se describen los principios generales y los compromisos del médico del siglo XXI, y se explicitan las exigencias que la sociedad actual le hace al médico en ejercicio, planteando los riesgos que existen actualmente para mantenerlo y, así, lograr que la sociedad apoye y fortalezca la autonomía de la profesión médica.

Se enfatiza que la autonomía que se defiende y se cree fundamental para un buen ejercicio de la medicina, es la autonomía de la profesión y no la autonomía individual del médico; de ahí que cobran importancia los protocolos y directrices que la profesión promulgue para orientar a sus afiliados. Finalmente, se esbozan algunos elementos de lo que puede hacer la profesión médica actualmente para mantener su autonomía.

PALABRAS CLAVE: profesionalismo, autonomía médica, compromiso del médico.

\section{SUMMARY}

The modern concept of professionalism and its application to medical practice is presented. From the medical professionalism project led by major American and European associations of Medicine, the basic principles and commitments of physicians on the XXI century and the demands that society makes to the medical practitioner are described, considering the risks that currently exist to maintain professionalism in current medical practice in order to achieve what society supports and strengthens the autonomy of the medical profession.

It is emphasized that the autonomy that is defended and is considered essential for a good medical practice is the autonomy of the profession and not the individual physician autonomy, for that reason protocols and guidelines promulgated by the medical profession become important to guide its affiliates. Finally, some elements of what medical profession can do now to maintain its autonomy are outlined.

KEY WORDS: Professionalism, medical autonomy, physician`s commitment.

\section{INTRODUCCIÓN}

El elemento central de una profesión es el trabajo basado en el dominio de un cuerpo de conocimientos y habilidades. Es una vocación en la que se utiliza el conocimiento de algún área de la ciencia al servicio de los demás. Sus miembros profesan un compromiso con la competencia científica y técnica, la integridad, la ética, el altruismo y

\section{Correspondencia:}

José María Maya

Email:

jmayam@ces.edu.co

Recibido: 5 de noviembre de 2015

Aceptado: 6 de noviembre de 2105

Conflictos de interés:

No se reportan conflictos de interés. 


\title{
“El concepto de profesión se define por la pertenencia de sus miembros a un cuerpo especializado de conocimiento, por su vocación de servicio y por su capacidad de autorregularse, y por lo tanto, organizar el modo en que se ofrecen sus servicios".
}

\author{
Richard Cruess y Sylvia Cruess'.
}

la promoción de un bien público dentro de su área. Estos compromisos constituyen la base del contrato social entre la profesión y la sociedad que, a su vez, otorga la autonomía profesional en la práctica y el privilegio de la autorregulación. Las profesiones y sus miembros son responsables ante la sociedad a la que deben rendir cuentas.

El contrato entre las profesiones y la sociedad es relativamente sencillo. La sociedad les concede a las profesiones un monopolio sobre la utilización de un conjunto de conocimientos, así como considerable autonomía, prestigio y retribución financiera, a cambio de la competencia profesional, la decisión de brindar un servicio altruista y llevar a cabo sus actividades de una manera ética e integra. La Medicina es una profesión, lo cual implica unas condiciones especiales, no solo en el proceso de formación, sino en su ejercicio. La profesión requiere de la adquisición y la aplicación de un conjunto de conocimientos y habilidades técnicas, y de una actitud especial frente al centro de su actuación: el paciente. Los miembros de la profesión están unidos por un compromiso compartido y la sociedad les ha otorgado la posibilidad de autorregularse.

El concepto de profesionalismo médico, catalogado como un competencia general por el Accreditation Council for Graduate Medical Education (ACGME) ${ }^{2}$, hace relación al conjunto de principios, valores, conocimientos y habilidades que sustentan la práctica médica con elevados estándares de calidad científica y ética. Es propio de la naturaleza de la Medicina y su tradición. El profesionalismo es el soporte del contrato de la medicina con la sociedad. Su base son los principios éticos, valores y conductas que sustentan el compromiso de los médicos con los pacientes y la sociedad ${ }^{3}$. Estos evolucionan y se replantean a la luz de los cambios sociales, y avalan la confianza que la sociedad tiene en sus médicos. Implica un deber hacia los pacientes, anteponiendo sus necesidades a los intereses del médico, asumiendo la responsabilidad de mantener los máximos estándares de competencia e integridad y la provisión de asistencia de alta calidad a la sociedad en materia de salud. En la sociedad del siglo XXI, que le ha dado una gran importancia a la autonomía del paciente, aparece como exigencia profesional el respeto por esta autonomía, lo que obliga al profesional, no solo a tomar buenas decisiones clínicas basadas en la mejor evidencia científica disponible, sino también, a comunicarse y dialogar con el paciente y aplicar las decisiones consensuadas con este, de una manera profesional, que signifique de verdad ayuda integral, aceptación de su mayoría de edad como ser humano (autonomía) y que tenga en cuenta el bien común.

Uno de los atributos del profesionalismo es un juicio independiente sobre cuestiones técnicas relacionadas con los conocimientos de su profesión. Lo anterior se relaciona con un concepto importante: la autonomía de la profesión médica a la que me referiré más adelante.

Cohen destaca que el profesionalismo da prioridad a un profundo sentido de lo que debe ser el médico, más que a lo que debe saber y hacer ${ }^{4}$.

\section{PROYECTO DE PROFESIONALISMO MÉDICO}

El proyecto de profesionalismo médico liderado por la American Board of Internal Medicine (ABIM), el American College of Physicians-American Society of Internal Medicine (ACP-ASIM) y la European Federation of Internal Medicine, 
tuvo como fruto la denominada Carta de Profesionalismo Médico, publicada en el 2002 en Annals of Internal Medicine $^{5}$, y a la que se han adherido más de 90 asociaciones de especialistas y subespecialistas de Estados Unidos. La carta se compone de una introducción que pone sobre el tapete el riesgo actual para el profesionalismo médico, generado en los cambios en los sistemas de salud y la pérdida creciente del compromiso del médico con la primacía del bienestar del paciente. El cuerpo central de la carta son los tres principios y los diez compromisos que fundamentan el profesionalismo médico en el siglo XXI.

\section{Los principios generales son los siguientes:}

- Primacía del Bienestar del Paciente. Los profesionales médicos deben dedicarse a servir a los mejores intereses del paciente, con altruismo y generando un alto nivel de confianza, lo cual es fundamental en la relación entre médico y paciente.

- Respeto por la AUtonomía del PACiente. Implica honestidad, entregar a ellos la posibilidad de tomar decisiones informadas sobre su cuidado (consentimiento informado) y orientación para evitar la atención inapropiada o innecesaria.

- Justicia social. La profesión debe promover la justicia en la atención de salud, evitando la discriminación de cualquier orden y contribuyendo a la distribución equitativa de los recursos destinados a la salud.

\section{Los diez compromisos son los siguientes:}

- Con las Competencias Profesionales. Implica compromiso de aprendizaje durante toda la vida laboral, y la responsabilidad de mantener el conocimiento, las habilidades clínicas y el equipo necesario para prestar una atención de calidad.

- Honestidad con los Pacientes. Implica suministrar información completa y honesta al paciente antes del consentimiento informado, y documentar los efectos secundarios y las fallas del tratamiento, como parte de un programa de seguridad del paciente y salvaguarda de la confianza.

- Confidencialidad. Consiste en mantener el secreto profesional y solo revelarlo en los casos previstos para defender el interés público o de terceros.

- Adecuadas Relaciones Con los PaCientes. Dada la vulnerabilidad y dependencia de los pacientes por la relación asimétrica generada con el profesional, hay ciertas relaciones que deben evitarse.
- Calidad en la atención. Implica el empeño por mejorar continuamente la calidad y mantener la competencia, trabajar para reducir el error médico, aumentar la seguridad del paciente, y optimizar la utilización de los recursos de salud y los resultados de la atención.

- Mejorar EL ACCESo a LA ATENCión. Implica trabajar para lograr la cobertura y disponibilidad de un buen estándar de atención para todos, eliminando las barreras de acceso y favoreciendo la promoción de la salud pública y preventiva.

- Distribución JUSTa de Recursos limitados. Implica proveer servicios de salud basados en análisis de costo-efectividad de los recursos clínicos; igualmente, el desarrollo de protocolos de atención basados en la evidencia científica, evitando procedimientos y exámenes innecesarios.

- Conocimiento científico. Conlleva el uso apropiado del conocimiento científico y la tecnología, la promoción de la investigación y la creación de nuevo conocimiento. La profesión es responsable por la integridad de este conocimiento, y que esté basado en evidencia científica y experiencia calificada.

- MANTENER LA CONFIANZA MANEJANDo LOS CONFLICTOS DE INTERÉs. Implica la obligación de reconocer, revelar y tratar los conflictos de interés que surgen en el curso de sus deberes y actividades profesionales, evitando poner en riesgo las responsabilidades personales o de sus organizaciones al relacionarse con industrias de insumos para la salud, compañías de seguros y otras.

- Responsabilidades profesionales. Se espera que los médicos trabajen en equipo para maximizar la calidad de la atención, tengan una buena relación con los colegas ('colegaje'), aceptando la corrección de los miembros que hayan fallado en el cumplimiento de los estándares profesionales, y participen en procesos de autorregulación, evaluación externa y escrutinio por terceros de su desempeño.

\section{EXIGENCIAS DE LA SOCIEDAD ACTUAL AL MÉDICO}

Las más importantes exigencias que la sociedad del siglo XXI le hace al profesional médico, son las siguientes.

- Vocación. Implica preeminencia del rol social por encima del beneficio económico.

- Disciplina. Es la subordinación individual del médico a los protocolos y reglas de la profesión. 
- Competencia. Es poseer conocimientos actualizados, buenas habilidades, y una positiva y respetuosa actitud.

- Compromiso. Consiste en brindar asistencia calificada al paciente, preocuparse por la salud de la comunidad y gestionar en forma eficiente los recursos para la salud.

\section{RIESGOS ACTUALES PARA MANTENER EL PROFESIONALISMO MÉDICO}

En el siglo XXI, el médico y el ejercicio de la medicina enfrentan conflictos y riesgos que amenazan sus principios y deterioran las relaciones entre el médico y el paciente, y entre la medicina y la sociedad. Esta situación está poniendo en riesgo el contrato social que la medicina tiene con la sociedad. Algunas de las situaciones que están poniendo en riesgo el profesionalismo médico, son:

- Pérdida de confianza de la sociedad en los profesionales ${ }^{6}$.

- Evidencias de deshumanización de los médicos.

- Desequilibrio creciente entre los recursos y las necesidades de salud.

- Creciente dependencia de las fuerzas del mercado para transformar y regular la prestación de los servicios de salud.

- Falta de autorregulación en la profesión y concepto equivocado de la autonomía profesional al entenderla como autonomía individual.

- Debilidad de los organismos colegiados representativos de la profesión?.

Hay un elemento preocupante. La autonomía de la profesión, que es parte de la contrapartida que la sociedad le da a cambio de su profesionalismo, está en riesgo. Tres supuestos sobre los que se sustentan la autonomía y el profesionalismo (maestría técnica, altruismo y autorregulación), se han debilitado, lo cual ha generado reacciones sociales e intromisión en la autonomía. Una hipótesis aceptada es que falló la autorregulación y sin ella no hay autonomía y sin esta se pone en riesgo el profesionalismo médico.

Lo anterior se acompaña de la alteración de la armonía entre la sociedad y la profesión, por varias de las diferentes razones expresadas anteriormente. Igualmente, se ha debilitado la transmisión del profesionalismo, como un modelo de conducta profesional, a los estudiantes de medicina y a la sociedad. Esta cada vez más duda del al- truismo de los médicos y los acusa de anteponer su propio beneficio, especialmente el económico, al de la sociedad.

\section{¿QUÉ PUEDE HACER LA PROFESIÓN?}

La única manera efectiva de mantener la fidelidad al contrato social de la profesión médica en la sociedad posmoderna, y en tiempos de crisis y cambios en los sistemas de salud, es reafirmando la observancia de los principios del profesionalismo. Si las asociaciones médicas, los profesionales y las instituciones de salud y educación médica, entendemos estos principios, los enseñamos y los cumplimos, podemos ir recuperando la autonomía de la profesión y la confianza de la sociedad.

Para que el profesionalismo médico sobreviva en la sociedad actual, es necesario y pertinente:

- Restablecer la racionalidad en el ejercicio profesional.

- Respetar el concepto de autonomía de la profesión, con lo que ello implica, y abandonar la defensa de la autonomía individual del profesional.

- Fortalecer el ejercicio profesional guiado por los valores de una ética civil.

- Redimensionar los privilegios y compensaciones que la sociedad da al médico, a cambio del cumplimiento de sus obligaciones.

- Restablecer las relaciones de la profesión con el Estado y con las otras profesiones.

- Enfatizar la enseñanza del profesionalismo en la educación médica.

En el siglo XXI, el ejercicio adecuado de la profesión se debe centrar en la autonomía del paciente, pero, sin olvidar que la profesión, y por ende los médicos, tenemos responsabilidades y deberes con la sociedad. Por esto, se requiere un gran esfuerzo para llegar a consensos que establezcan sobre qué bases operacionales prácticas se apoyarán los estándares del profesionalismo para su aplicación en el ejercicio diario, como una manera de recuperar, mantener y conservar la confianza del ciudadano en la profesión, como un todo, y en sus médicos, en forma individual. Igualmente, es necesario que todo médico comprenda claramente los orígenes y la naturaleza de su condición profesional, y las obligaciones necesarias para sostenerla. El profesionalismo debe ser enseñado ${ }^{8}$ en forma explícita para que, teniendo un conocimiento claro de él, sea adoptado como modelo que se debe seguir. 


\title{
“Ni los incentivos económicos, ni la tecnología, ni el control administrativo, han demostrado ser sustitutos efectivos del compromiso con la integridad evocado en el ideal del profesionalismo".
}

\author{
William Sullivan.
}

Las asociaciones médicas deben adoptar unas normas de conducta a tono con los principios del profesionalismo, lo que conlleva el ser extremadamente prudentes en la forma de negociar para sus miembros. Cualquier indicio de que el bien público está siendo ignorado, es perjudicial para la credibilidad de la profesión y el resultado es la pérdida de la confianza en ella. Si no hay transparencia en su actuación y la asociación aparece como quien encubre un ejercicio inadecuado de la profesión, el precio que pagaremos será muy alto. Si la profesión lleva acciones en contra del bien común y solo nos ven defender nuestros propios intereses, es probable que perdamos la posibilidad de ejercer con base en un verdadero profesionalismo.

Igualmente, el sistema de salud debe promover activamente un comportamiento deseable de los profesionales médicos, para evitar que pongamos nuestro propio interés en primer lugar o que defendamos una autonomía individual en el ejercicio profesional, aislándonos de la profesión como colectivo autorregulador, o que ignoremos la autonomía del paciente en la relación o abandonemos el compromiso con la salud pública y el uso razonable, justo y eficiente de los recursos que el sistema ha destinado para todos los ciudadanos.

\section{CONCLUSIÓN}

Se hace necesario reconstruir el profesionalismo médico a partir de una clarificación común entre los profesionales y la sociedad, lo cual será de beneficio mutuo. Las academias y los colegios médicos deben ser el vehículo para impulsar el profesionalismo, con el apoyo de las escuelas de Medicina. El desafío está en fortalecer la profesión (el ser) como prerrequisito para mejorar la ocupación (el hacer). Es hacer lo que la sociedad espera de los profesionales médicos (altruismo, competencia, respeto por la autonomía, aceptación de la primacía del bien común) para lograr lo que queremos, que es vivir la profesión a plenitud y como fuente de paz interior y felicidad, y no solo ocuparnos de ella. Si la profesión se guía por los valores y principios del profesionalismo, la sociedad terminará entendiendo que es bueno para ella y la apoyará.

\section{REFERENCIAS}

1. Cruess R, Cruess S. El credencialismo como garantía social en el contexto social. Relaciones con el profesionalismo médico. En: Bosh A, Alenta E, editores. La profesión médica: los retos del milenio. Barcelona: Fundación Medicina y Humanidades Médicas; 2004. p. 33-46.

2. Accreditation Council for Graduate Medical Education. Competencias generales. Chicago: ACGME; 1999. Fecha de consulta: 5 de octubre de 2015. Disponible en: http://www.acgme.org/ acWebsite/home/home.asp.

3. Patiño JF. El profesionalismo médico. Rev Colomb Cir. 2004;19:146-52.

4. Cohen JJ. Teoría de las profesiones. La medicina como paradigma de profesión preeminente. En: Bosh A, Alenta E editores. La profesión médica: los retos del milenio. Barcelona: Fundación Medicina y Humanidades Médicas; 2004. p. 5-10.

5. Medical professionalism in the new millennium. A physician charter. Project of the ABIM Foundation, ACP Foundation, European Federation of Internal Medicine. Ann Intern Med. 2002;136:243-6.

6. Pellegrino E. Trust and distrust in professional ethics. En: Pellegrino E, Veath R, Langan J, editors. Ethics, trust and the professions: Philosophical and cultural aspects. Washington, D.C.: Georgetown University Press; 1991. p. 69-85.

7. Pellegrino E, Relman A. Professional medical associations: Ethical and practical guidelines. JAMA. 1999;282:984-6. Fecha de consulta: 2 de octubre de 2015. Disponible en: http://jama. ama-assn.org/content/282/10/984.full.pdf + html.

8. Cruess R, Cruess S. Professionalism must be taught. BMJ. 1997;315:1674-7.

9. Sullivan W. El credencialismo como garantía social en el contexto social. Relaciones con el profesionalismo médico. En: Bosh A, Alenta E editores. La profesión médica: los retos del milenio. Barcelona: Fundación Medicina y Humanidades Médicas; 2004. p. 5. 